\title{
On the Q-switched operation of Titanium:Sapphire lasers using a graphene-based saturable absorber mirror
}

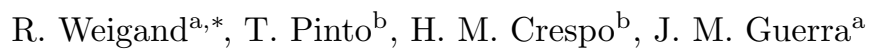 \\ ${ }^{a}$ Departamento de Óptica. Facultad de Ciencias Físicas. Universidad Complutense de \\ Madrid. Avda. Complutense s/n. 28040 Madrid. SPAIN \\ ${ }^{b}$ Departamento de Física e Astronomia e IFIMUP-IN, Faculdade de Ciências, Universidade \\ do Porto, R. do Campo Alegre 687, 4169-007 Porto, Portugal
}

\begin{abstract}
We numerically demonstrate Q-switched operation of Titanium:Sapphire lasers using mono and multilayer graphene, deposited on a totally reflecting end mirror as a saturable absorber. Output energies, pulse duration and repetition frequencies of the Q-switched pulse trains are given as a function of the pump intensity for different number of graphene layers and cavity lengths. For the geometries studied, pulses from 16.2 to $467 \mathrm{~ns}$ can be achieved, with energies ranging from 8.7 to $71 \mu \mathrm{J}$ and repetition rates from 0.057 to $2.27 \mathrm{MHz}$. These results can be useful for designing and building laser cavities for Q-switched and mode-locked operation in laser media with short lifetimes as Titanium:Sapphire.
\end{abstract}

Keywords: Q-switch, Ti:S laser, Graphene-based saturable absorber

\section{Introduction}

In recent years, graphene has been successfully employed as saturable absorber for Q-switched and mode-locked operation in a variety of lasers with different gain media. Mode-locked operation in fibers has been obtained [1, 2] 5 as well as in bulk media such as laser rods [3, 4, 5, 6, Q. Q-switched operation delivers longer but more energetic pulses, which can be useful in many applications such as materials processing, laser ranging, LIDAR, ultrafast laser

*Corresponding author: weigand@fis.ucm.es 
pumping, medicine, etc. This temporal regime has been achieved in fiber lasers [7, 8, 9, 10, 11, 12, waveguides [13] and laser rods [14, 15, 16, 17, 18, 19, 20, 21, 22, 23, 24. With these results, the use of monolayer and multilayer graphene as saturable absorber in laser cavities seems to be well controlled from the experimental point of view. However it is well known that the mode-locking regime can coexist with the Q-switched regime and this combination is not always desirable, since mode-locking appears within a "Q-switched envelope", which gives Q-switched operation in a certain laser medium for a specific cavity design could be of great interest to avoid undesired designs. Due to its broadband absorption characteristics, graphene is a particularly interesting material for ultrashort pulse generation by saturable absorber mode-locking in lasers with broadband emission such as the Titanium:sapphire laser. There are already reports on mode-locking of Titanium:Sapphire lasers using monolayer graphene [5]. Regarding the apparition of the Q-switched regime using this laser material, it is generally known that, given the short lifetime of Titanium:Sapphire $(3.2 \mu \mathrm{s}), \mathrm{Q}-$ switched operation with fast-saturable absorbers is not possible. However some authors have reported it $25,26,27$. In this paper we show that it is possible to obtain Q-switched operation in Titanium:Sapphire lasers using graphene as a saturable absorber. Stable Q-switching is observed and studied for cavity lengths between 500 and $110 \mathrm{~mm}$, with mode-locked pulse repetition rates from $300 \mathrm{MHz}$ to $1.36 \mathrm{GHz}$, respectively. These values are typical of high-repetition rate broadband Ti:Sapphire laser oscillators, which have important applications in time-resolved [28] and frequency-domain 29] spectroscopies. The majority of these lasers rely on Kerr-lens mode-locking, which is normally not self-starting. The possibility of employing broadband saturable absorption, such as provided by graphene, as a reliable and self-starting mode-locking mechanism is therefore 35 highly appealing. The results presented in this work show that the potential occurrence of simultaneous Q-switching must be taken into account in the study and design of broadband saturable absorption mode-locked high-repetition rate lasers even in media with short emission lifetimes such as Ti:Sapphire. 


\section{Numerical simulation}

40 known process [30, 31]. Being $t_{r}$ the cavity roundtrip time; $t_{L}, t_{A}$ the relaxation times of the laser medium and absorber respectively; $l$ the cavity losses and $P_{L}$, $P_{A}$ the saturation power of the laser medium and absorber, then the intracavity power $P$, the gain $g$ and the density of population inversion $q$ can be calculated 45 with the following set of equations [30, 31]:

$$
\begin{gathered}
d P / d t=1 / t_{r}(g-l-q) P \\
d g / d t=-1 / t_{L}\left(\left(g-g_{0}\right)+g P / P_{L}\right) \\
d q / d t=-1 / t_{A}\left(\left(q-q_{0}\right)+q P / P_{A}\right),
\end{gathered}
$$

where the meaning of $g_{0}$ and $q_{0}$ is explained below.

Using this approach we could successfully reproduce the experimental results obtained with a Nd:YLF laser using a monolayer G-SAM (Graphene deposited on a mirror) [19] by solving the set of equations using a 4-th order Runge-Kutta method.

In this study we have chosen a linear cavity (Fig. 1) with a $4.5 \mathrm{~mm}$ long Ti:Sapphire rod with Brewster-cut edges, which is representative of typical 10 fs systems. We considered a thermal lens of $10 \mathrm{~mm}$ focal length in the rod and a refractive index of 1.78. A lens L1 with $10 \mathrm{~mm}$ focal length is used to focus the laser beam onto the G-SAM and was placed at $18 \mathrm{~mm}$ from it. Another lens L2 with $50 \mathrm{~mm}$ focal length was placed next to the output coupler OC to stabilize the cavity. The distance D2 from the left-hand crystal face to lens L2 was 50 $\mathrm{mm}$ and the distance D3 from L2 to the OC was changed to have different total cavity lengths, while the distance D1 from the right-hand crystal face to lens L1 was kept at $24 \mathrm{~mm}$. The total optical length from lens L2 to the G-SAM was $100 \mathrm{~mm}$. To compensate for the astigmatism produced by the Brewster-cut 
faces of the crystal, both lenses were tilted an angle of $3.5^{\circ}$. The stability of the cavity and the waist sizes of the laser beam were calculated using gaussian beam propagation. The waist at the laser rod $w_{L}$ and at the G-SAM, $w_{G}$, are given in Table I. We see that $w_{G}$ changes a factor of 2 for the cavity lengths chosen. The values given are an average between the values in the sagittal and tangential planes. The optical paths of the different cavities were $L=110,200$, 300, 400 and $500 \mathrm{~mm}$. The average reflectivities given by $R=\sqrt{R_{1} R_{2}}$ with $R_{1}$ and $R_{2}$ the reflectivities of the end mirror (99.8\%) and output coupler $(97 \%)$ respectively. Internal cavity losses were taken as $\alpha_{i} L=0.135$, which is a value within the typical order of magnitude for solid state lasers.

The cavity losses are given by $l=2\left(\alpha_{i} L-\ln (R)\right)$. The quantity $q_{0}$ is the reflectivity change in the end mirror from absorbing to saturated graphene. Since a single graphene layer absorbs $\sim 2.3 \%$, the absorbance of $n$ graphene layers ${ }_{75}$ is $q_{0}=1-0.977^{2 n}$. The value of $g_{0}$ is given by $g_{0}=2 \alpha_{0} * L=I / I_{t h}\left(\alpha_{i} L-\ln (R)\right)$, were $g_{0}$ depends on the pumping intensity above threshold, and not on the threshold value itself.

Saturation powers for the absorber or the laser medium $P_{\text {sat }}=P_{A}, P_{L}$ were calculated from the saturation fluence $F_{\text {sat }}$. We used $P_{\text {sat }}=F_{\text {sat }} \pi w^{2} / \tau$, with $\tau$ the relaxation time of the laser medium $\left(t_{L}=3.2 \mu \mathrm{s}[32]\right)$ or of graphene $\left(t_{A}=1.45 \mathrm{ps}\right.$ [4] $), w$ the beam waists at the laser rod $\left(w_{L}\right)$ or at the G-SAM $\left(w_{G}\right)$, which, as mentioned, were estimated in the stability analysis (Table 1). The saturation fluence for Titanium:Sapphire is given by $F_{\text {sat }}=h \nu /(2 \sigma)$, with $h$ Planck's constant, $\nu$ the laser emission frequency and $\sigma$ the emission cross 85 section $\left(\sigma=4.1 \times 10^{-19} \mathrm{~cm}^{2}\right.$ for the electric field parallel to the c-axis for Ti:S 32. For graphene, $F_{\text {sat }}$ is $14.5 \mu \mathrm{J} / \mathrm{cm}^{2}$ [4].

By solving equations 1 to 3 using a 4-th order Runge-Kutta method, we have studied the Q-switched regime (pulse duration, pulse energy and repetition frequency of the Q-switch train) as a function of the pump intensity above 90 threshold $\left(I / I_{t h}\right)$, for different cavity lengths and different number of layers. The integration time was always set long enough so that the stable regime (constant peak power) was reached. The results can be seen in Figs. 2 to 7 . Figure 
2 shows the temporal shape of typical Q-switched pulse trains obtained with these calculations. It is worth noting that there are two temporal behaviors of the Q-switch pulses obtained. One with zero cw background and another with a certain cw component, depending on the pump intensity. The cases shown correspond to two cases with $L=500 \mathrm{~mm}$, and since the pump intensity is different repetition rates are also different.

Some general behaviors can be deduced from the information contained in Figs. 3 to 7. Pulse duration corresponds to the FWHM (Full Width Half Maximum), the pulse energy was obtained by integrating the output power $\left(1-R_{2}\right) P$ in time, and the repetition frequency is the inverse of the temporal separation of two consecutive pulses. All magnitudes were obtained by averaging the values of the last four pulses obtained in the simulations for times long enough to have reached a stationary state. The pulse duration and the pulse energy in this regime were calculated by subtracting the cw background where necessary. Missing points in the graphs for certain pump intensities $I$ mean that no QSwitched regime is obtained (for instance, cw regime or relaxation oscillations are observed instead). Given a certain cavity length, the pulse duration and the energy per pulse increase with the number of graphene layers, while the repetition frequency decreases with the number of layers. This is because the reflectivity change $q_{0}$ of the G-SAM is larger for a larger number of layers. On the other hand, when the cavity length increases, the pulse duration increases, the pulse energy diminishes and the repetition frequency remains approximately the same. The cavity length $L$ has a direct influence on the cavity losses $l$ which increase when $L$ increases. With high cavity losses the system cannot build short pulses and also cannot deliver pulses with high energy. However the repetition frequency is not altered since the repetition frequency in the Q-switched regime does not depend on the length of the cavity, as it does in the mode-locking regime, but it is rather governed by the lifetimes of the saturable absorber and the laser medium. For all the cavity lengths and for the different number of graphene layers the dependency of pulse duration, pulse energy and repetition frequency on the pump intensity $I$ is the one expected for a laser operation 
in Q-switched regime. When the pump intensity grows above threshold the intracavitary power will be higher, so the saturable absorber can be bleached for a longer time, which means that the pulse will be longer. It can also be bleached to a larger extent, so the energy delivered per pulse will be higher. And finally, after a pulse has been emitted, if the pump intensity is higher the system can bleach the saturable absorber faster, so another pulse can rapidly build up and hence the repetition frequency increases. However in those cases in which the Q-switched operation appears above the cw background, the energy per pulse decreases with pump intensity (Fig. 7) since the power carried by cw background grows more rapidly.

\section{Conclusions}

35 In conclusion, by employing a standard theory for a Q-switched operation of lasers we have demonstrated Q-switched operation of a Ti:S laser using a G-SAM as saturable absorber for different cavity lengths ranging from $110 \mathrm{~mm}$ to $500 \mathrm{~mm}$. Depending on the cavity length, the number of graphene layers and the pump intensity, pulses with $16.2 \mu$ s to $467 \mu$ s duration have been obtained, with energies from $8.7 \mu \mathrm{J}$ to $71 \mu \mathrm{J}$. The repetition rates of the Q-switched pulse trains extended from $0.057 \mathrm{MHz}$ to $2.27 \mathrm{MHz}$.

The results obtained show mainly two things. On one side they show that graphene can effectively be used as a saturable absorber to obtain Q-switched regime with laser materials with a short emission lifetime. On the other hand they can help to properly design laser cavities where Q-switching should be avoided to favor mode-locked operation. This should be particularly important for the shorter cavity lengths which are typical of high-repetition rate (usually $\mathrm{GHz}$ or multi-GHz) lasers.

\section{Acknowledgements}

This work was partially supported by project Santander-UCM PR6/1318875. H. Crespo acknowledges grant PTDC/FIS/122511/2010 from Fundaçâo 
para a Ciência e Tecnologia, Portugal, co-funded by COMPETE and FEDER.

Support from the European Science Foundation through the SILMI programme (Super-intense laser-matter interactions), grant 6658 , is gratefully acknowledged. mund, Efficient mode-locking of sub-70-fs ti:sapphire laser by graphene saturable absorber, Appl. Phys. Express 5 (3) (2012) 032701.

URL http://stacks . iop.org/1882-0786/5/i=3/a=032701

[6] S. C. Xu, B. Y. Man, S. Z. Jiang, D. J. Feng, S. B. Gao, C. S. Chen, M. Liu, 口 C. Yang, C. Zhang, D. Bi, F. Y. Liu, X. Meng, Sapphire-based graphene 
saturable absorber for long-time working femtosecond lasers, Opt. Lett.

39 (9) (2014) 2707-2710. doi:10.1364/OL.39.002707.

URL http://ol.osa.org/abstract.cfm?URI=0l-39-9-2707

[ [7] Z. Luo, M. Zhou, J. Weng, G. Huang, H. Xu, C. Ye, Z. Cai, Graphenebased passively q-switched dual-wavelength erbium-doped fiber laser, Opt. Lett. 35 (21) (2010) 3709-3711. doi:10.1364/0L.35.003709.

URL http://ol.osa.org/abstract.cfm?URI=0l-35-21-3709

口 [8] D. Popa, Z. Sun, T. Hasan, F. Torrisi, F. Wang, A. C. Ferrari, Graphene

a q-switched, tunable fiber laser, Appl. Phys. Letters 98 (7) (2011) -. doi: http://dx.doi.org/10.1063/1.3552684. URL http://scitation.aip.org/content/aip/journal/apl/98/7/10. $1063 / 1.3552684$

q [9] M. Han, S. Zhang, X. Li, H. Zhang, F. Wen, Z. Yang, High-energy, tunable-wavelengths, q-switched pulse laser, Opt. Commun. 326 (0) (2014) 24 - 28. doi:http://dx.doi.org/10.1016/j.optcom.2014.04.012. URL http://www.sciencedirect.com/science/article/pii/ S0030401814003460

[10] N. Kasim, C. L. Anyi, H. Haris, F. Ahmad, N. M. Ali, H. Ah-

a mad, Y. Munajat, S. W. Harun, Q-switched erbium-doped fiber laser using multi-layer graphene based saturable absorber, J. Non200 ㄴ linear Opt. Phys. Mater. 23 (01) (2014) 1450009. arXiv:http: //www.worldscientific.com/doi/pdf/10.1142/S021886351450009X, doi:10.1142/S021886351450009X.

(1) URL http://www.worldscientific.com/doi/abs/10.1142/ S021886351450009X

${ }_{205}^{2}$ [11] F. D. Muhammad, M. Z. Zulkifli, H. Ahmad, Graphene based q-switched tunable s-band fiber laser incorporating arrayed waveguide gratings (awg),

口 J. Nonlinear Opt. Phys. Mater. 23 (01) (2014) 1450004. arXiv:http: //www.worldscientific.com/doi/pdf/10.1142/S0218863514500040, 


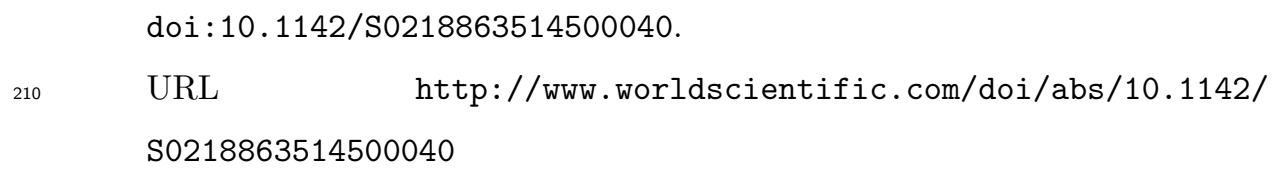

[12] Y. Tang, X. Yu, X. Li, Z. Yan, Q. J. Wang, High-power thulium fiber laser q-switched with single-layer graphene, Opt. Lett. 39 (3) (2014) 614-617. doi:10.1364/0L.39.000614

URL http://ol .osa.org/abstract . cfm?URI=ol-39-3-614

[13] Y. Tan, C. Cheng, S. Akhmadaliev, S. Zhou, F. Chen, Nd:yag waveguide laser q-switched by evanescent-field interaction with graphene, Opt. Express 22 (8) (2014) 9101-9106. doi:10.1364/0E.22.009101.

口 URL http://www.opticsexpress.org/abstract.cfm?URI= oe-22-8-9101

[14] M. Jiang, Z. Ren, Y. Zhang, B. Lu, L. Wan, J. Bai, Graphene-based passively q-switched diode-side-pumped nd:yag solid laser, Opt. Commun.

(1) 284 (22) (2011) 5353 - 5356. doi:http://dx.doi.org/10.1016/j. optcom.2011.07.063

[15] H. Yu, X. Chen, X. Hu, S. Zhuang, Z. Wang, X. Xu, J. Wang, H. Zhang, M. Jiang, Graphene as a q-switcher for neodymium-doped lutetium vanadate laser, Appl. Phys. Express 4 (2) (2011) 022704. URL http://stacks .iop.org/1882-0786/4/i=2/a=022704

[16] X. lei Li, J. long $\mathrm{Xu}, \mathrm{Y}$. zhong $\mathrm{Wu}$, J. liang He, X. peng Hao, a Large energy laser pulses with high repetition rate by graphene $\mathrm{q}$ switched solid-state laser, Opt. Express 19 (10) (2011) 9950-9955. doi:10.1364/OE.19.009950

235

URL http://www.opticsexpress.org/abstract.cfm?URI= oe-19-10-9950 
[17] Q. Wang, H. Teng, Y. Zou, Z. Zhang, D. Li, R. Wang, C. Gao, J. Lin, L. Guo, Z. Wei, Graphene on sic as a q-switcher for a $2 \mu \mathrm{m}$ laser, Opt. Lett. 37 (3) (2012) 395-397. doi:10.1364/0L.37.000395. URL http://ol .osa.org/abstract . cfm?URI=ol-37-3-395

[18] G. Q. Xie, J. Ma, P. Lv, W. L. Gao, P. Yuan, L. J. Qian, H. H. Yu, H. J. Zhang, J. Y. Wang, D. Y. Tang, Graphene saturable absorber for q-switching and mode locking at $2 \mu \mathrm{m}$ wavelength, Opt. Mater. Express 2 (6) (2012) 878-883. doi:10.1364/OME.2.000878.

URL http://wWw.opticsinfobase.org/ome/abstract.cfm?URI= ome-2-6-878

[19] P. Matía-Hernando, J. M. Guerra, R. Weigand, An nd:ylf laser q-switched by a monolayer-graphene saturable-absorber mirror, Laser Phys. 23 (2) (2013) 025003. URL http://stacks . iop.org/1555-6611/23/i=2/a=025003

[20] C. J. Jin, X. M. Chen, L. F. Li, M. Qi, Y. Bai, Z. Y. Ren, J. T. Bai, A 1. graphene-based passively q -switched ho:yag laser in-band pumped by a diode-pumped tm:ylf solid-state laser, Laser Phys. 24 (3) (2014) 035801. URL http://stacks . iop .org/1555-6611/24/i=3/a=035801 C. Zhang, D. Bi, X. Meng, F. Liu, Watt-level passively q-switched

घ mode-locked yvo4/nd:yvo4 laser operating at $1.06 \mu \mathrm{m}$ using graphene as a saturable absorber, Opt. Laser Tech. 56 (0) (2014) 393 - 397. doi:http://dx.doi.org/10.1016/j.optlastec.2013.09.028. URL http://www.sciencedirect.com/science/article/pii/ S0030399213003538

[22] R. P. Shi, Y. Bai, M. Qi, X. M. Chen, H. D. Wei, Z. Y. Ren, J. T. Bai, A 1. passively mode-locked intracavity frequency doubled nd:yvo 4 femtosecond green laser based on graphene, Laser Phys. Lett. 11 (2) (2014) 025001.

URL http://stacks . iop.org/1612-202X/11/i=2/a=025001 
[23] S. D. D. D. Cafiso, E. Ugolotti, A. Schmidt, V. Petrov, U. Griebner, A. Agnesi, W. B. Cho, B. H. Jung, F. Rotermund, S. Bae, B. H.

n Hong, G. Reali, F. Pirzio, Sub-100-fs cr:yag laser mode-locked by monolayer graphene saturable absorber, Opt. Lett. 38 (10) (2013) 1745-1747.

URL http://ol .osa.org/abstract . cfm?URI=ol-38-10-1745

[24] M. N. Cizmeciyan, J. W. Kim, S. Bae, B. H. Hong, F. Rotermund, A. Sennaroglu, Graphene mode-locked femtosecond cr:znse laser at $2500 \mathrm{~nm}$, Opt. Lett. 38 (3) (2013) 341-343. doi:10.1364/OL.38.000341. URL http://ol.osa.org/abstract.cfm?URI=0l-38-3-341

[25] Q. Xing, W. Zhang, K. Yoo, Self-q switched self-mode-locked ti: $\quad$ sapphire laser, Opt. Commun. 119 (12) (1995) 113 - 116. doi:http://dx.doi.org/10.1016/0030-4018(95)96930-2.

I URL http://www.sciencedirect.com/science/article/pii/ 0030401895969302

[26] E. Sali, E. Ignesti, S. Cavalieri, L. Fini, M. Tognetti, R. Buffa, A tuneable,

n single-mode titanium-doped-sapphire laser source with variable pulse duration in the nanosecond regime, Opt. Commun. 282 (16) (2009) 3330 3334. doi:http://dx.doi.org/10.1016/j.optcom.2009.05.014. S0030401809004660

[27] E. Sali, E. Ignesti, S. Cavalieri, L. Fini, M. Tognetti, R. Buffa, A titanium1 d doped-sapphire laser source with tunable frequency, single-mode emission,

口 and adjustable pulse duration, Laser Phys. 20 (5) (2010) 1126-1131. doi: 10.1134/S1054660X10090136.

URL http://dx.doi.org/10.1134/S1054660X10090136

[28] A. Bartels, T. Dekorsy, H. Kurz, Femtosecond ti\&\#x003a;sapphire ring व laser with a 2-ghz repetition rate and its application in time-resolved spec- 
troscopy, Opt. Lett. 24 (14) (1999) 996-998. doi:10.1364/OL.24.000996.

[29] C.-H. Li, A. J. Benedick, P. Fendel, A. G. Glenday, F. X. Kartner, D. F.

1

[31] H. Haus, Parameter ranges for cw passive mode locking, IEEE J. Quantum Electron. 12 (3) (1976) 169-176. doi:10.1109/JQE.1976.1069112.

[32] W. Koechner, Solid-State Laser Engineering, New York: Springer, 2006. 
Table 1: Size of the beam waists at the laser rod and at the G-SAM.

\begin{tabular}{ccc}
\hline Cavity length & $\begin{array}{c}\text { Beam waist } \\
\text { at the laser rod }\end{array}$ & $\begin{array}{c}\text { Beam waist } \\
\text { at the G-SAM }\end{array}$ \\
\hline$(\mathrm{mm})$ & $(\mu \mathrm{m})$ & $(\mu \mathrm{m})$ \\
110 & 61.2 & 28.9 \\
200 & 54.2 & 23.0 \\
300 & 51.9 & 19.6 \\
400 & 51.1 & 16.9 \\
500 & 56.0 & 14.1 \\
\hline
\end{tabular}




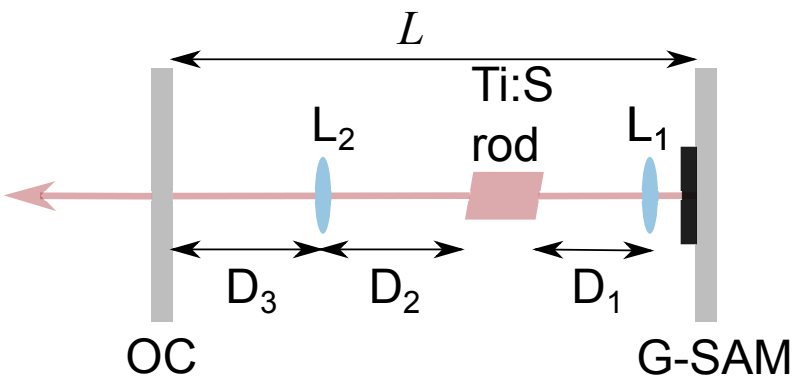

Figure 1: (colour online) Cavity design. FL: Focusing Lens, OC: Output Coupler, G-SAM: Graphene Saturable Absorber Mirror. L1, L2: lenses, D1 - D3: distances between marked elements $L$ : cavity length. 


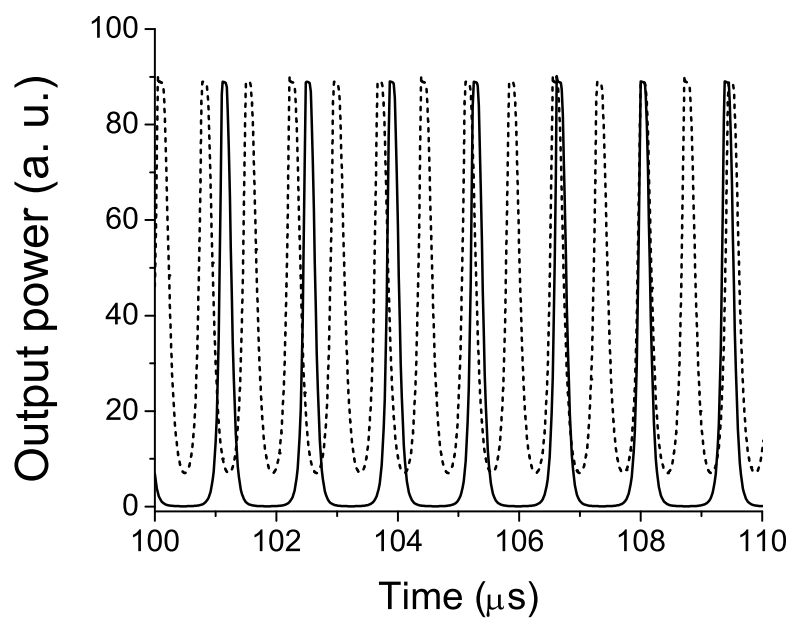

Figure 2: Temporal behaviour of typical Q-switched pulse trains obtained in the simulations without cw background (solid line) and with cw background (dashed line). 


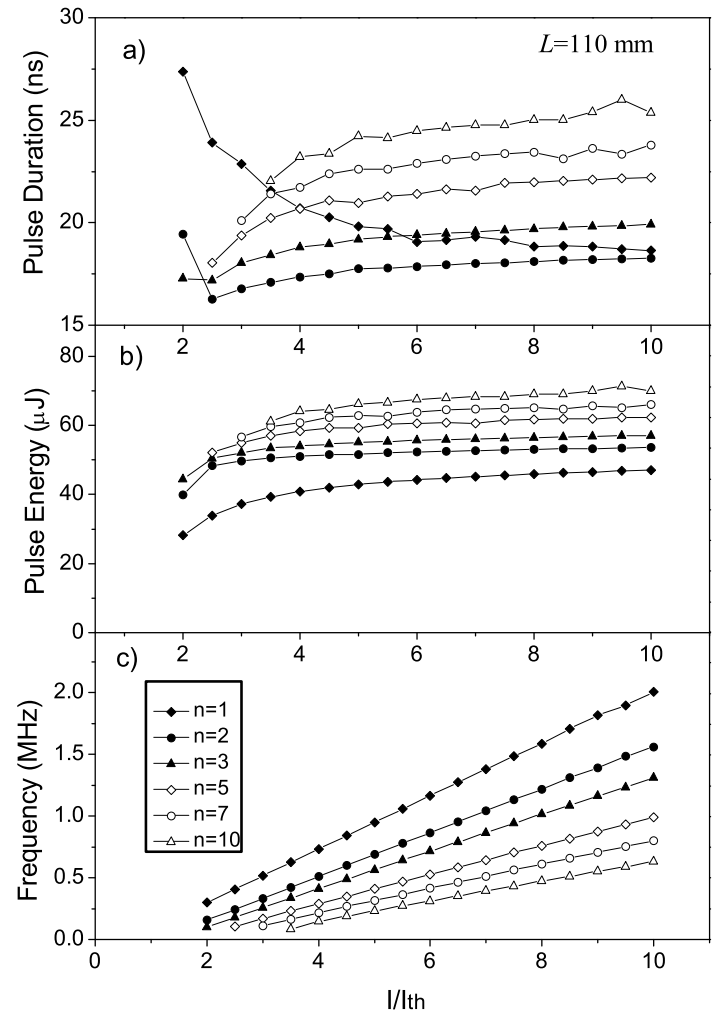

Figure 3: (a) Pulse duration, (b) pulse energy and (c) repetition frequency of the Q-switched pulse trains as a function of $I / I_{t h}$ and for different number of graphene layers $(\bullet \mathrm{n}=1, \bullet \mathrm{n}=2$, $\Delta \mathrm{n}=3, \diamond \mathrm{n}=5, \circ \mathrm{n}=7, \triangle \mathrm{n}=10)$. Cavity length $L=110 \mathrm{~mm}$. 


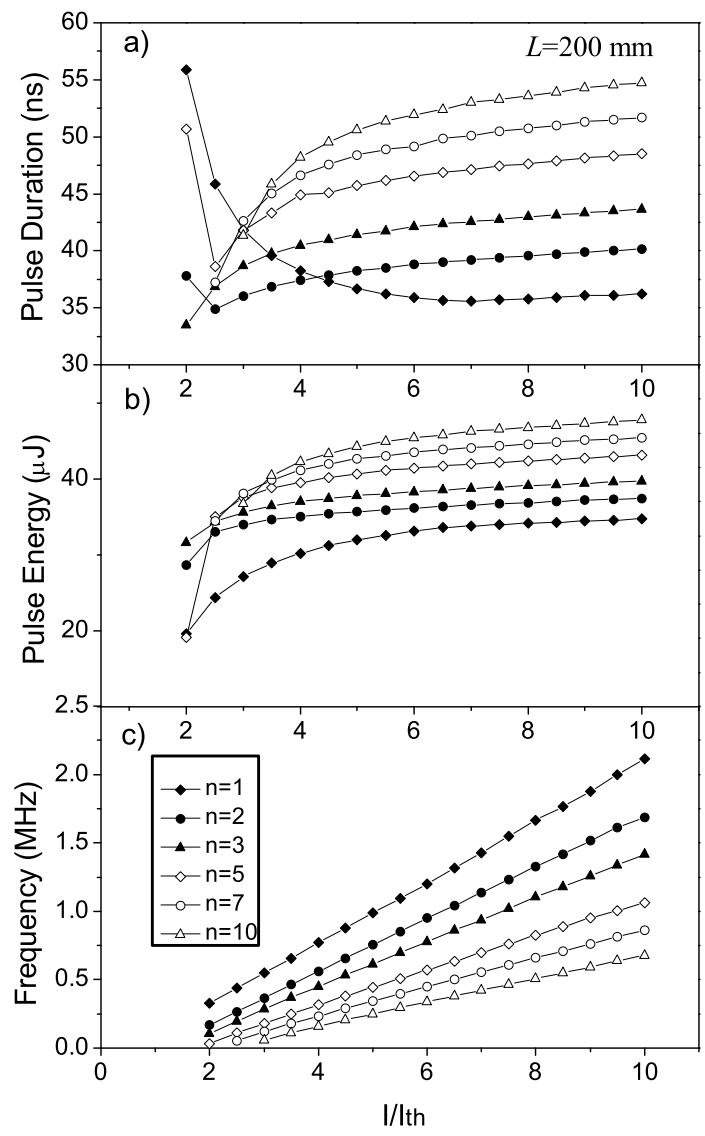

Figure 4: (a) Pulse duration, (b) pulse energy and (c) repetition frequency of the Q-switched pulse trains as a function of $I / I_{t h}$ and for different number of graphene layers $(\bullet \mathrm{n}=1, \bullet \mathrm{n}=2$, $\Delta \mathrm{n}=3, \diamond \mathrm{n}=5, \circ \mathrm{n}=7, \Delta \mathrm{n}=10)$. Cavity length $L=200 \mathrm{~mm}$. 


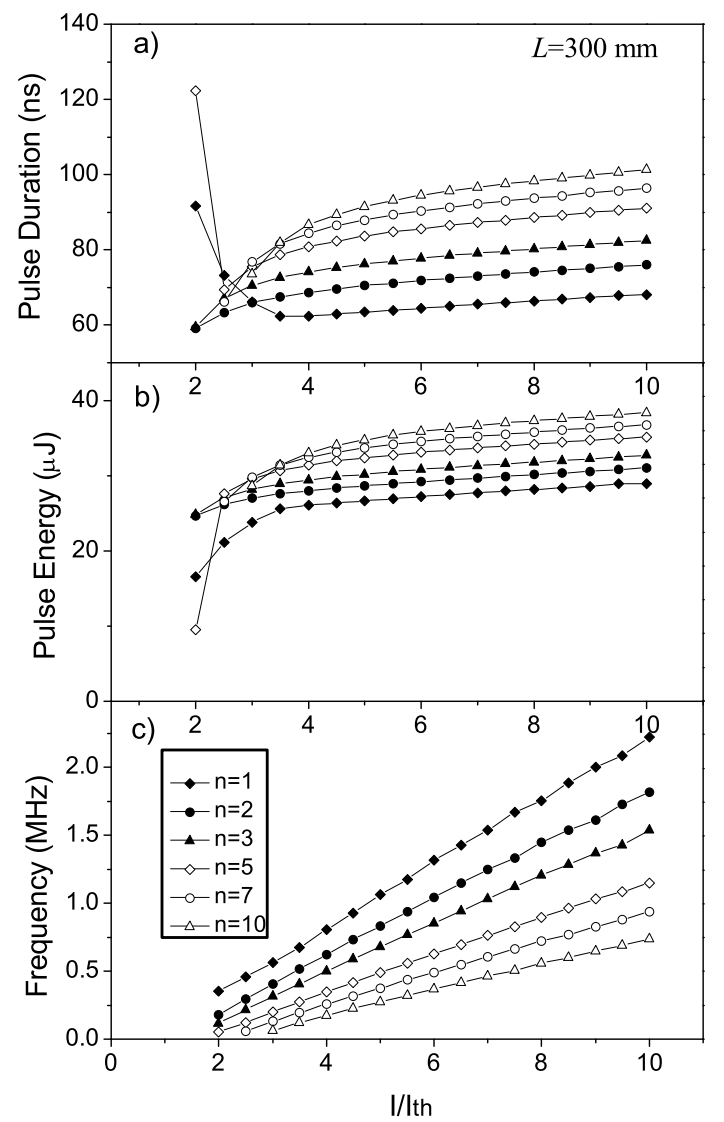

Figure 5: (a) Pulse duration, (b) pulse energy and (c) repetition frequency of the Q-switched pulse trains as a function of $I / I_{t h}$ and for different number of graphene layers $(\bullet \mathrm{n}=1, \bullet \mathrm{n}=2$, $\Delta \mathrm{n}=3, \diamond \mathrm{n}=5, \circ \mathrm{n}=7, \triangle \mathrm{n}=10)$. Cavity length $L=300 \mathrm{~mm}$. 


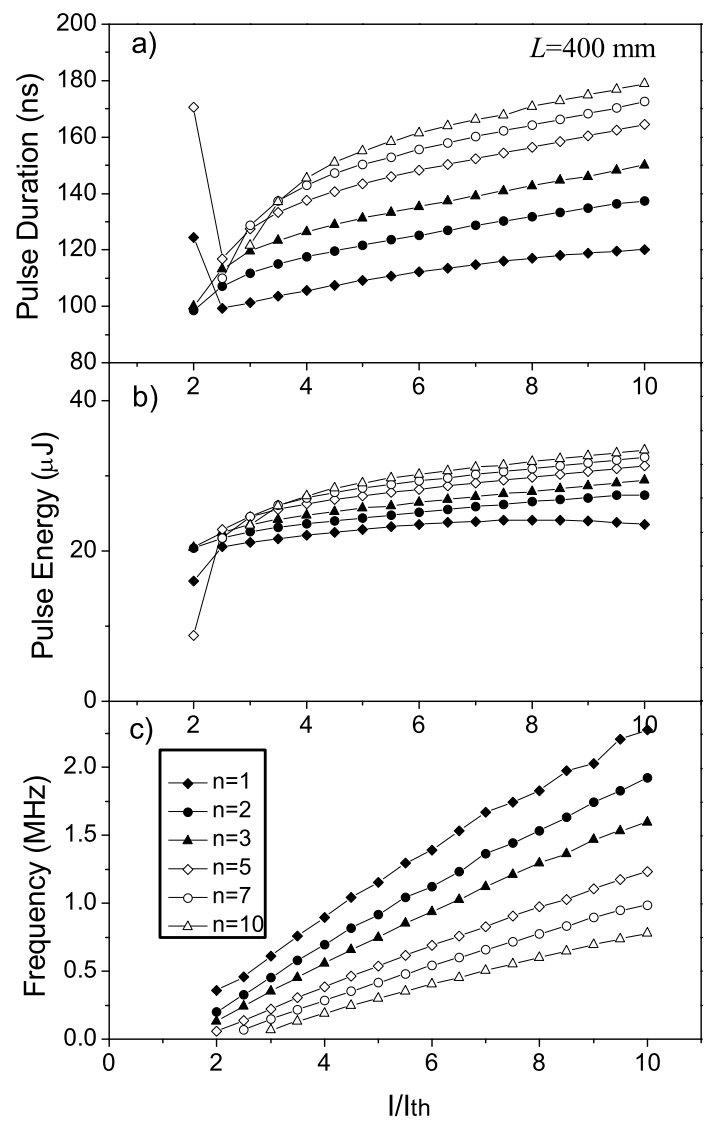

Figure 6: (a) Pulse duration, (b) pulse energy and (c) repetition frequency of the Q-switched pulse trains as a function of $I / I_{t h}$ and for different number of graphene layers $(\bullet \mathrm{n}=1, \bullet \mathrm{n}=2$, $\Delta \mathrm{n}=3, \diamond \mathrm{n}=5, \circ \mathrm{n}=7, \triangle \mathrm{n}=10)$. Cavity length $L=400 \mathrm{~mm}$. 


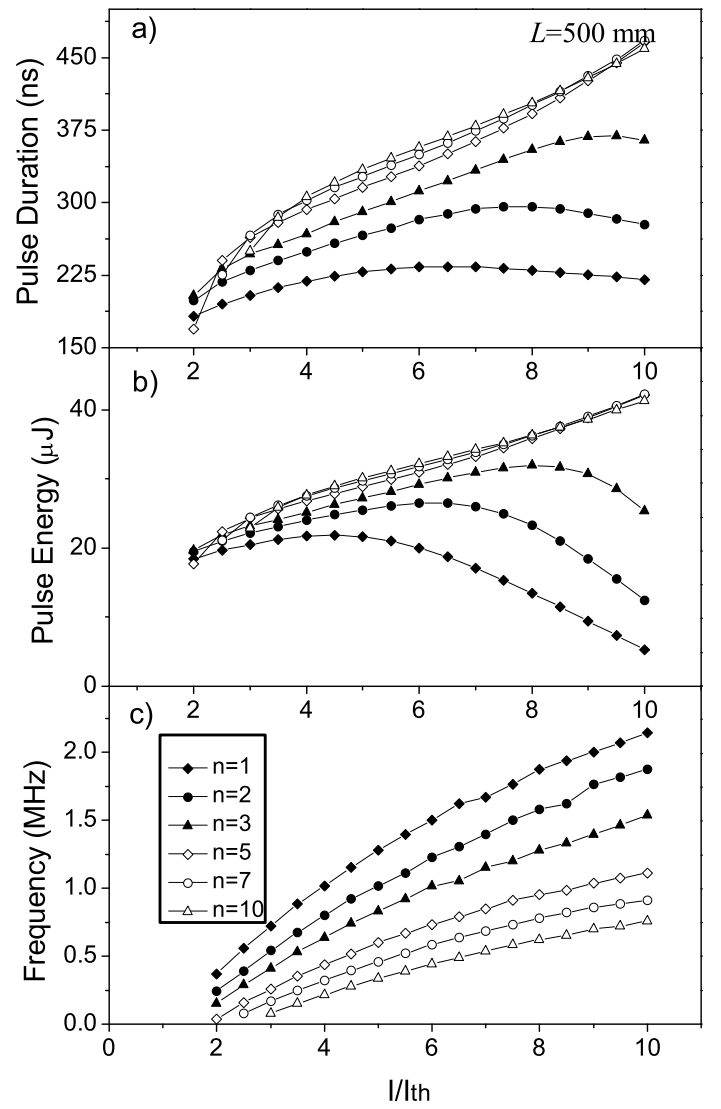

Figure 7: (a) Pulse duration, (b) pulse energy and (c) repetition frequency of the Q-switched pulse trains as a function of $I / I_{t h}$ and for different number of graphene layers $(\bullet \mathrm{n}=1, \bullet \mathrm{n}=2$, $\Delta \mathrm{n}=3, \diamond \mathrm{n}=5, \circ \mathrm{n}=7, \triangle \mathrm{n}=10)$. Cavity length $L=500 \mathrm{~mm}$. 


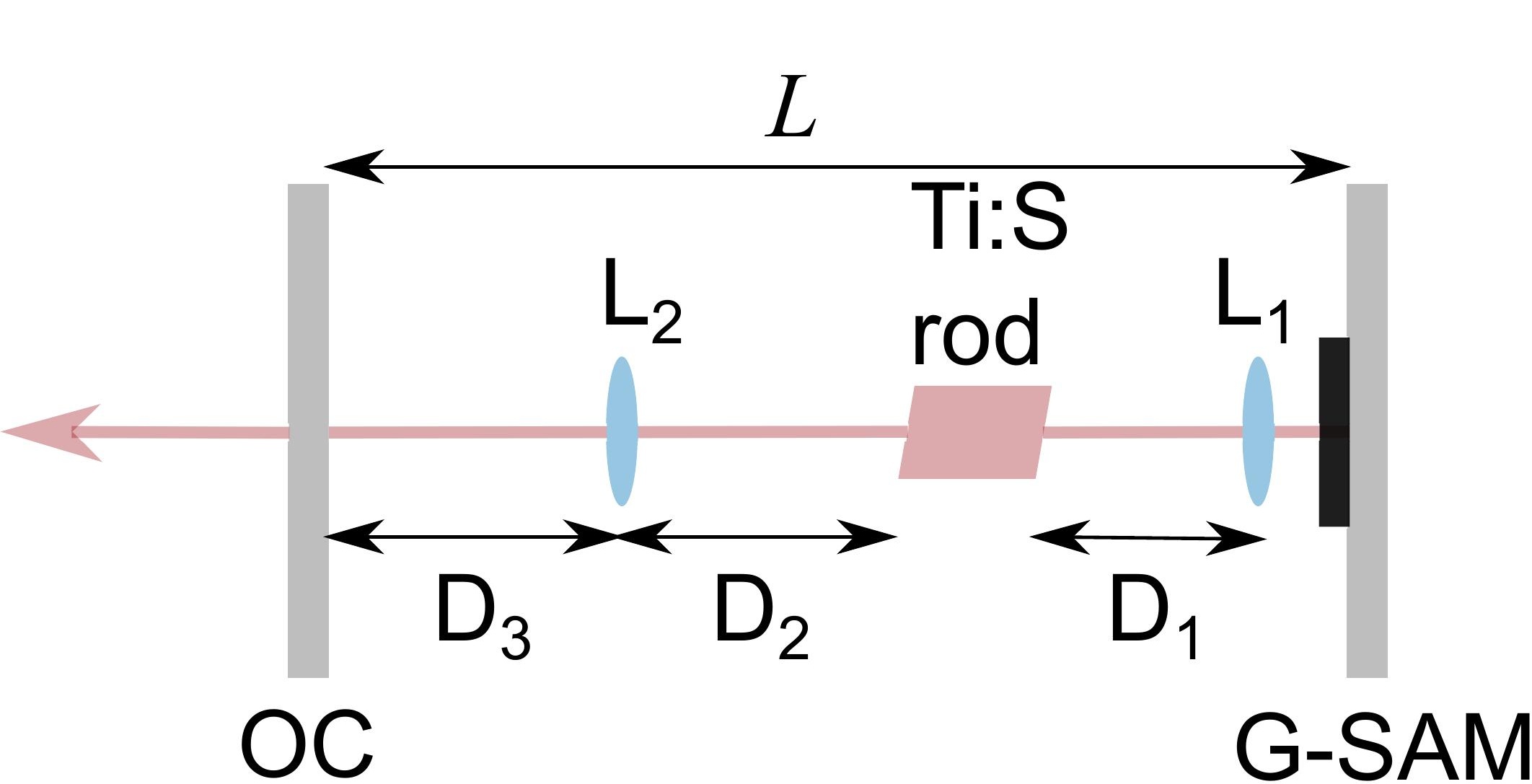




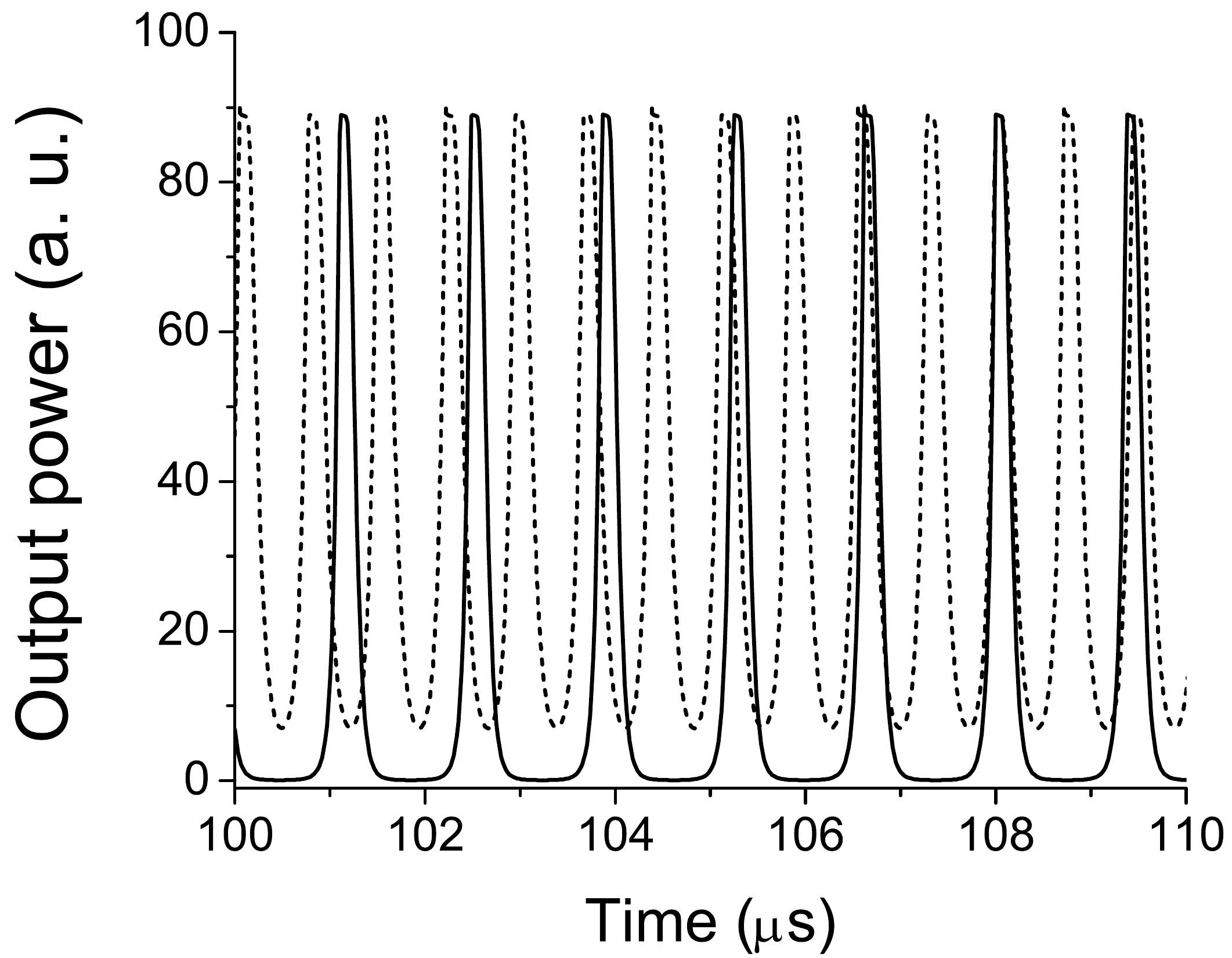




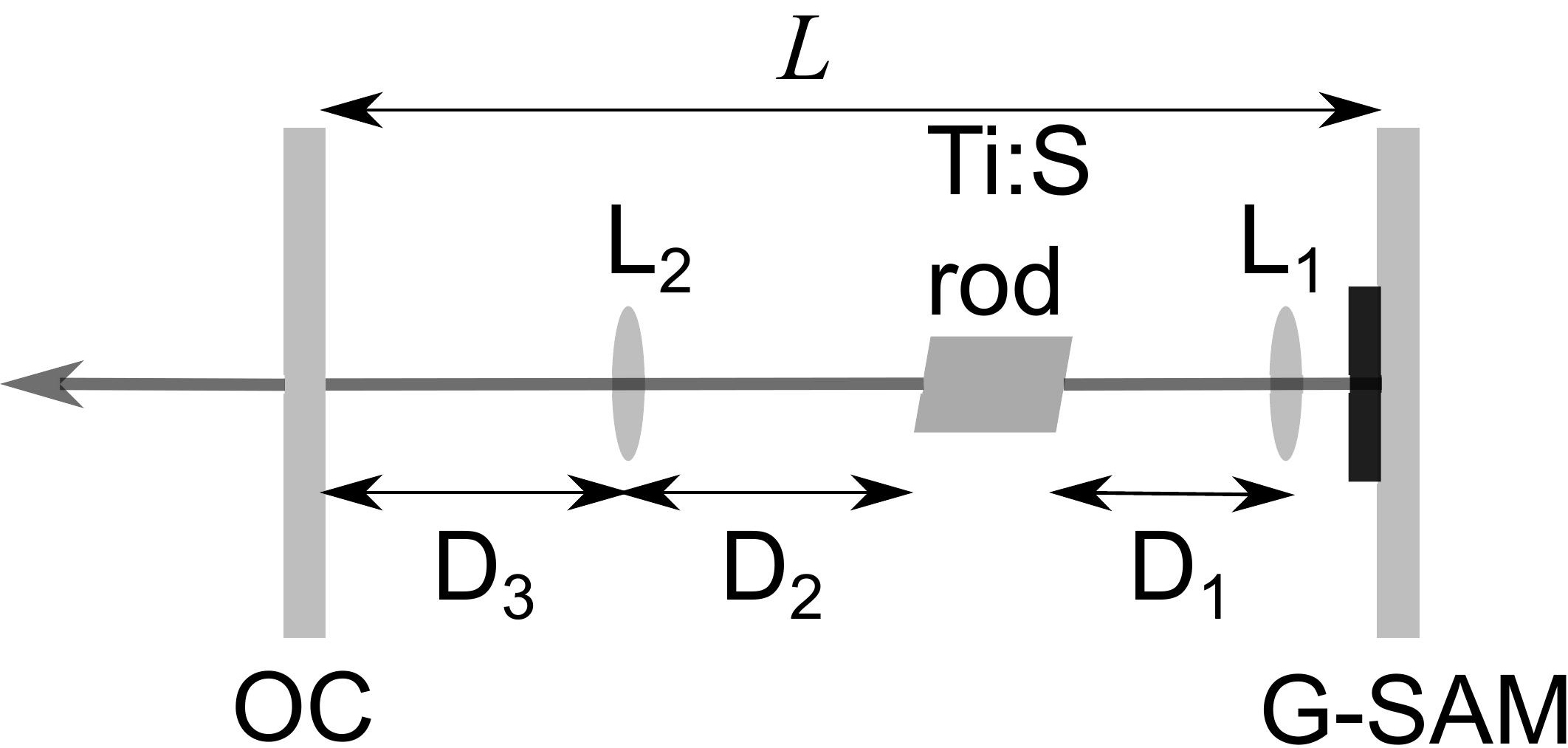

Figure 